The Psychological Record, 2009, 59, 75-92

\title{
ESTABLISHING DERIVED MANDING FOR SPECIFIC AMOUNTS WITH THREE CHILDREN: AN ATTEMPT AT SYNTHESIZING SKINNER'S VERBAL BEHAVIOR WITH RELATIONAL FRAME THEORY
}

\author{
Carol Murphy and Dermot Barnes-Holmes \\ National University of Ireland, Maynooth, Ireland
}

\begin{abstract}
Participants were 2 typically developing children, aged 9 and 10 years, and 1 child, aged 4 years, with a reported severe speech delay. Five specific mand functions were trained such that participants learned to mand for the delivery or removal of tokens to the value of $-2,-1,0,+1$, and +2 , by presenting an arbitrary stimulus (A1, A2, A3, A4, and A5, respectively). The A stimuli were then incorporated into a series of interrelated conditional discriminations (A1-B1, B1-C1, A2-B2, B2-C2, A3-B3, B3-C3, A4-B4, B4-C4, A5-B5, B5-C5). Subsequent tests determined if participants derived 5 specific mands, presenting $C 1, C 2, C 3$, $C 4$, and $C 5$ as mands for $-2,-1,0,+1$ and +2 tokens. Three participants demonstrated derived manding, and derived manding altered in accordance with newly trained relations across two reversal procedures.
\end{abstract}

Many researchers have suggested that the study of derived stimulus relations may help to provide a functional-analytic account of human language and higher cognition (Barnes-Holmes, Barnes-Holmes, \& Cullinan, 2000; Hayes, Barnes-Holmes, \& Roche, 2001). The untrained or "emergent" nature of derived relations seems especially relevant to the "generativity" that is characteristic in human language. Specifically, humans are frequently observed to create, combine, and understand novel sentences that they have never heard before. Explaining such novel verbal responding is important to a behavior analytic theory of language, because it has long been criticized for failing to deal with the issue of generativity (Chomsky, 1959).

One example of generativity in the derived stimulus relations literature is the derived transfer of functions. This effect is typically observed across two stages. First, participants are trained and tested in a series of interrelated conditional discriminations that give rise to equivalence relations (e.g., A-B-C). Second, a particular function is established for one of the stimuli in the relation (e.g., A is trained as a discriminative stimulus, or Sd), and this function then

The current study was partially funded by the Research and Graduate Studies Department at NUI Maynooth. The Irish Research Council for the Humanities and Social Sciences also provided partial funding for the research.

All correspondence should be addressed to Carol Murphy at the Department of Psychology, National University of Ireland, Maynooth, Co. Kildare, Ireland. E-mail: Carol.A.Murphy@nuim.ie 
emerges for the other stimuli without direct training (e.g., C acquires an Sd function in the absence of differential reinforcement). Derived transfer effects have been well documented in the basic experimental literature (e.g., Barnes, Browne, Smeets, \& Roche, 1995; Dougher, Auguston, Markham, Greenway, \& Wulfert, 1994), and recently derived transfer of a verbal request or mand function (Skinner, 1957) has been demonstrated in an applied setting with participants in whom autism has been diagnosed (Murphy, Barnes-Holmes, \& Barnes-Holmes, 2005; Rehfeldt \& Root, 2005; Rosales \& Rehfeldt, 2007; Murphy \& Barnes-Holmes, in press).

Skinner's argument for distinct verbal operants, and in particular the "mand" operant, has proved beneficial in applied settings when teaching children with autism or language deficits, or both (Addison, Vorndren, Volkert, \& Kodak, 2005; Lerman, Parten, Sundberg \& Michael, 2001; Sundberg \& Partington, 1998). A "mand" is a verbal operant that frequently specifies its own reinforcer (e.g., "give me milk" or "stop shouting"), and learning to mand effectively may facilitate a novel form of indirect environmental control via a social mediator or "listener" (Sundberg \& Michael, 2001). The development of a mand repertoire may therefore provide a speaker with improved control over his or her environment and facilitate access to reinforcers and the removal of punishers.

The simplest technique for training mands involves directly reinforcing specific mands under appropriate conditions of deprivation and frequently involves the use of motivating operations (MOs) to alter the momentary effectiveness of particular reinforcers. The term motivating operation (Laraway, Snycerski, Michael, \& Poling, 2003; Michael, 1993) refers to the manipulation of variables to alter the value of reinforcers and evoke increased frequency of responding previously associated with the specific reinforcer. An example of an MO might be arranging mild water deprivation when training a child to ask for water.

In attempting to establish a mand repertoire in a teaching context, it may be useful to distinguish between mands that have been directly taught and those that have not. Indeed, Skinner referred to untrained or novel mands as "magical mands," for example, highly generalized mands that are unlikely to receive reinforcement, as in "A horse, a horse, my kingdom for a horse!" However, Skinner's framework remained largely focused on directly trained mands and recombination of these, and conditions giving rise to novel manding were not specified in detail: "The speaker appears to create new mands on the analogy of old ones. ... Having successfully manded bread and butter, he (the speaker) goes on to mand the jam, even though he has never before obtained jam in this way (Skinner, 1957, p. 48).

Recently, researchers have integrated Skinner's concept of the "mand" with derived relations (Barnes-Holmes, Barnes-Holmes, \& Cullinan, 2000) and have begun to examine how novel mands emerge without direct training in an applied setting (Murphy et al., 2005; Rehfeldt \& Root, 2005; Rosales \& Rehfeldt, 2007; Murphy \& Barnes-Holmes, in press). In a study involving children with autism, a repertoire of derived mands for "more" items was successfully established (Murphy et al., 2005). Participants were first trained to mand for one of two tokens by presenting one of two stimulus cards for each type of token. The stimulus cards were then incorporated into two sets of conditional discriminations with other stimulus cards (A1-B1, B1-C1, A2B2, B2-C2). Participants were subsequently exposed to a test to determine 
whether the specific mand functions established for A1 and A2, respectively, would emerge for $\mathrm{C} 1$ and $\mathrm{C} 2$, respectively, in accordance with the trained conditional discriminations. Correct manding with $\mathrm{C} 1$ and $\mathrm{C} 2$ in the absence of differential consequences was deemed a demonstration of derived manding.

This study of derived manding was extended by follow-up research, again involving children with autism, that successfully established derived manding for the removal, as well as the addition, of tokens (Murphy \& BarnesHolmes, in press). This study first established "more" and "less" relations for two stimulus cards (A1 and A2). Training in conditional discriminations followed, and participants learned to relate these two stimuli with other stimuli (A1-B1, B2-C1, A2-B2, B2-C2). Subsequent tests were conducted to probe for derived more/less manding based on the trained conditional discriminations. All 4 participants successfully demonstrated manding with $\mathrm{C} 1$ for more tokens and manding with $\mathrm{C} 2$ for fewer tokens without direct training (i.e., in the absence of differential reinforcement or explicit instruction).

The study of derived manding in applied settings could be important in general terms, because relatively few studies have examined untrained manding (Hall \& Sundberg, 1987; Lamarre \& Holland, 1985; Nuzzolo-Gomez \& Greer, 2004; Petursdottir, Carr, \& Michael, 2005; Sigafoos, Reichle, Doss, Hall, \& Pettitt, 1990; Sundberg, San Juan, Dawdy, \& Arguelles, 1990; Twyman, 1996). In extending the work on derived manding, therefore, it will be important to develop procedures that serve to establish increasingly complex derived mands. Doing so could help to establish mand repertoires that more fully overlap with advanced mand repertoires found in the general population. The current study sought to extend the previous work by focusing on a relatively complex mand repertoire.

Although the previous studies developed procedures that successfully established untrained mand responses, the mands were relatively simple and involved asking for the addition or removal of a single stimulus item (Murphy et al., 2005; Murphy \& Barnes-Holmes, in press). Relatively complex mand repertoires, however, are frequently observed among sophisticated speakers. For example, mand responses are often quite precise, in that each of a series of mands may specify a particular quantity, such as "May I have one/two/three?" etc. Given the increased control that this level of verbal precision permits the speaker, it seems important that appropriate procedures are developed to establish such skills. To examine derived manding, one objective might be to establish a series of derived mand responses, each of which specifies the addition or removal of a particular number of stimulus items.

The current study sought to establish five specific derived mands with 3 children, 2 of whom were typically developing, and 1 of whom had a reported speech delay. Procedures commenced with training five specific mand functions for stimuli (i.e., mand for $-2,-1,0,+1$, and +2 , by presenting $A 1$, A2, A3, A4, and A5, respectively). The stimuli were then incorporated into interrelated conditional discriminations (A1-B1, B1-C1, A2-B2, B2-C2, A3-B3, B3-C3, A4-B4, B4-C4, A5-B5, B5-C5). Tests followed to determine whether five derived mand functions emerged for the $C$ stimuli (would the specific mands trained with the A stimuli subsequently emerge for the $C$ stimuli?). Reversal procedures were subsequently conducted to demonstrate experimental control of derived manding. The 3 participants ultimately demonstrated 
derived manding and a double reversal of derived manding. The findings are discussed in relation to establishing complex derived manding.

\section{General Method}

\section{Participants}

Ben, aged 9 years, attended a school for typically developing children and had no reported learning difficulties. Jasmine, aged 10, also attended a "mainstream" school but had remedial classes for language and maths for 2 hours per week. Shane, aged 4, had Applied Behavior Analysis tuition programs to remediate a severe speech delay and stereotypical behavior reported by his parents. None of the participants had been previously exposed to matchingto-sample (MTS) procedures involving arbitrary stimuli such as nonsense syllables used in the current study. All 3 participants could (minimally) add and subtract single-digit numbers up to the level used in the current study $(-2,-1,0,+1,+2)$.

\section{Experimental Setting}

The experimental procedures were conducted in the children's private homes. A desk and two chairs were used in all experimental phases, with participants seated on one side, facing the investigator, who was seated on the opposite side. For all test phases, a third chair was placed to one side of the desk (out of direct eye contact with the child), and the investigator moved to this chair while a parent sat in the investigator's chair. The parent conducted all tests and remained unaware of experimental objectives, while the investigator simply functioned as a second data recorder for the purposes of interrater agreement. Procedures were thus designed to prevent the possibility of subtle cueing.

The 3 participants performed all training and test trials individually, and sessions were usually conducted three or four times a week with each child. Sessions were always interspersed with frequent 2- to 5-min breaks during which participants could engage in a different activity of their choice. The number of sessions required for participants to complete the experiment ranged from 9 to 22 across participants. The duration of sessions was never more than $1 \mathrm{hr}$, and if a child showed any sign of distress or boredom, sessions were terminated and resumed at a later date.

\section{Materials}

All materials were prepared with laminated card stock that was colored to make it attractive. The stimuli used for mand training and testing and conditional discriminations consisted of 15 nonsense syllables printed on laminated cards (10 by $6 \mathrm{~cm}$; see Table 1). In the current report, the syllables are referred to as A1, A2, A3, A4, A5, B1, B2, B3, B4, B5, C1, C2, C3, C4, and C5; participants were never exposed to these labels. Mand training and testing also involved a rectangular board $(30 \mathrm{~cm}$ by $20 \mathrm{~cm}$ ) that had a center panel outlined in black containing six circles, each of which was also outlined in black, on which tokens were placed during training and test procedures. Tokens used throughout the experiment were 8-cm disks with printed smiley faces. 
Table 1

Nonsense Syllables Used Throughout Experimental Procedures

\begin{tabular}{ccccc}
\hline NIP & BEM & RUF & LOA & ICT \\
GAF & MEE & ZIV & POG & LUH \\
YEP & UNT & IBE & TRA & SEV \\
\hline
\end{tabular}

\section{Interrater Agreement}

Interrater agreement was obtained by having the investigator and a parent both record data independently across all test procedures for each participant. Interrater agreement was calculated by dividing the total agreements by the number of agreements plus disagreements and multiplying by 100\%. Agreement was calculated for all tests and was 100\% across participants.

\section{Procedure}

Mand training. To "evoke" manding for specific amounts of tokens, a board-game format was designed. In effect, this might be described as a conditioned MO (see Laraway et al., 2003; Michael, 1993). An MO refers to the manipulation of setting events (e.g., deprivation to increase the effectiveness of a mand consequence) and is a procedure used frequently in mand training. The board-game format involved the addition or removal of tokens being established as a conditioned reinforcer for participants by using praise and "points" for "winning a game." This was done using a token board containing six circles that had to be filled with six tokens. Specifically, participants were required to mand with particular stimulus cards for the addition or removal of specific amounts of tokens to retain a set of six in the center panel. Each mand stimulus card had a different printed nonsense syllable (see Table 1). (Studies involving derived transfer frequently use nonsense syllables or arbitrary symbols to prevent confounding influences due either to physical similarities between stimuli or to participants' learning histories outside the experimental context.) During training, the mands were reinforced, but during testing there were no programmed differential consequences (see Figure 1 for a visual representation of the procedural sequence).

The token board was placed on the desk in front of participants, and an array of mand stimulus cards (A1, A2, A3, A4, and A5) was placed in random order approximately $12 \mathrm{~cm}$ below (Figure 2A). The investigator told participants that the center panel of the board should be filled with six tokens. On a trial in which the child was required to mand for additional tokens, the investigator placed fewer than six tokens on the circles in the center panel. On a trial in which the child was required to mand for the removal of tokens, the investigator covered each of the six circles with a token and placed additional tokens in the space outside the panel. Finally, on a trial in which the child was required to mand for neither the addition nor the removal of tokens, the 
six circles were each covered by a token and no additional tokens were placed outside the panel. Thus, empty circles in the center panel served as visual prompts for absent tokens, and the outline of the panel emphasized that tokens present outside the line were deemed to be surplus (i.e., more than were needed).

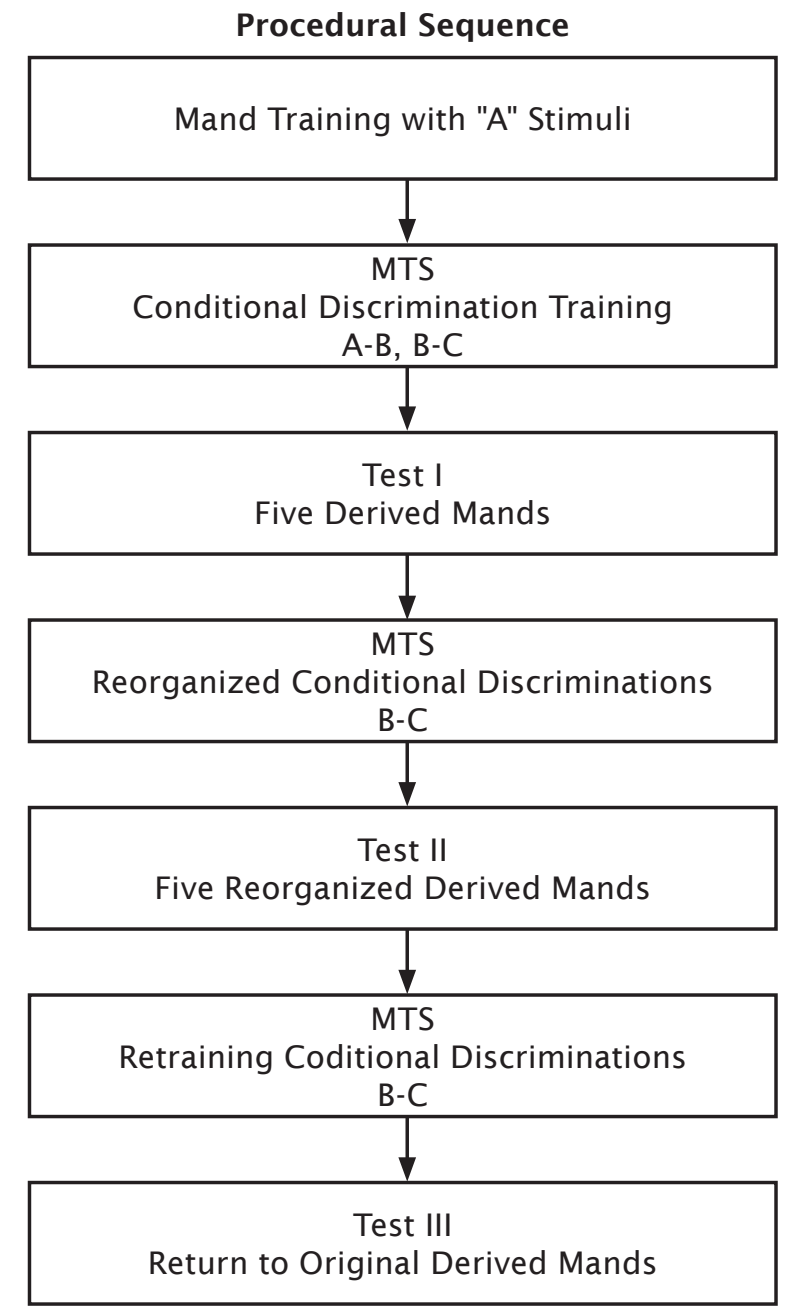

Figure 1. Sequence of training and test procedures.

Participants were instructed to mand to gain or lose specific amounts of tokens by presenting one of the five mand stimuli (A1, A2, A3, A4, and A5) to the investigator. The amount of tokens required might be $-2,-1,0,+1$, or +2 , and the participant had to mand for these specific amounts with A1, A2, A3, A4, or A5, respectively. Thus, for each trial, the investigator placed an amount of tokens $(8,7,6,5$, or 4$)$ on the board, and participants had to mand for correct amounts to make six tokens. Initially, mand training trials presented participants with the mands A1 and A2 only; thus the investigator placed either seven or eight tokens on the board, saying, 
Let's play a game. Look at the board and count the tokens-you must have six tokens here (pointing to the center). If there are eight tokens, like now, give me this card (A1) to lose two tokens. If there are seven tokens, give me this card (A2) to lose one token.

The investigator delivered positive reinforcement for "correct" manding (i.e., manding with A1 when presented with eight tokens on the board, or manding with A2 when there were seven tokens on the board). Positive reinforcement involved delivery of one "point" (points system), frequently paired with verbal praise (e.g., "That's right" or "Well done"). Points were later used to access backup reinforcers such as toys, games, activities, or edibles, and participants were allowed to have a 2- to 5-min break at this time.

If a participant manded "incorrectly," the investigator delivered no positive reinforcement, presented or removed the (incorrect) number of tokens manded, delivered a correction, cleared the board, and began the next trial. For example, if a participant manded with A2 (the mand for -1 token) when presented with eight tokens on the board, the investigator removed one token, saying, "You should have given me this card (pointing to A1) because you needed to lose two tokens." The trial was recorded as incorrect, the board cleared, and the investigator moved on to the next trial.

As participants became proficient at manding with A1 or A2 (four correct consecutive trials), the investigator gradually introduced manding with A3, A4, and A5 across the next six trials. The position in which the five mand stimuli were presented, in a horizontal line from left to right, was held constant across initial trials (see block-trial procedure, Smeets \& Striefel, 1994). When all five mand stimuli had been introduced (approximately 10 trials), the positional prompt was removed and the position of mand stimuli was randomly rotated across subsequent trials. Mand trials were counted in blocks of 25, and these included only trials involving all five mand stimuli with random positioning. Presentation of mand training trials was arranged in a quasi-random order, but each 25-trial block involved 5 trials for each of the mand stimuli (A1, A2, A3, A4, and A5). The criterion for successful completion of mand training was a minimum of $22 / 25$ correct responses for two successive blocks.

Conditional discrimination training. When participants had successfully completed mand training with the "A" stimuli, an MTS procedure was used to train the following interrelated conditional discriminations with the A stimuli and other arbitrary stimuli (nonsense syllables): A1-B1, B1-C1, A2-B2, B2-C2, A3-B3, B3-C3, A4-B4, B4-C4, A5-B5, B5-C5. On each trial, the investigator positioned one of the A stimuli (samples) on the desk in front of the participant, approximately $14 \mathrm{~cm}$ above five comparison stimuli (B1, B2, B3, B4, and B5) arranged in random order. For initial trials, the investigator gave a simple instruction to participants (e.g., when the sample was A1, "Look here [A1], and point here [B1]," and when the sample was A2, "Look here [A2], and point here [B2]"). As trials continued the investigator placed the sample and said, "Which one should you point to?," and eventually simply placed the sample and waited for participants to point.

If a participant made a correct selection, the investigator delivered positive reinforcement (as per mand training). If a participant made an incorrect selection, the investigator gave a correction indicating the "B" stimulus to be selected and delivered no positive reinforcement. Initially the investigator used 
2A.Mand Training Example

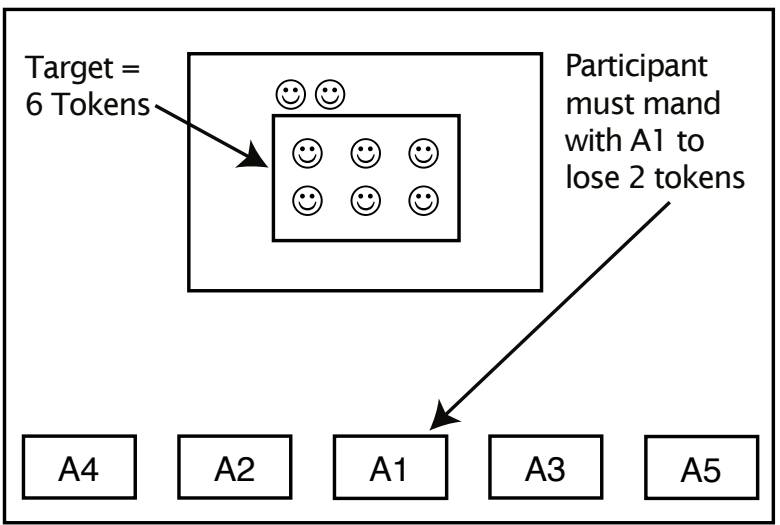

2B.Test I Example

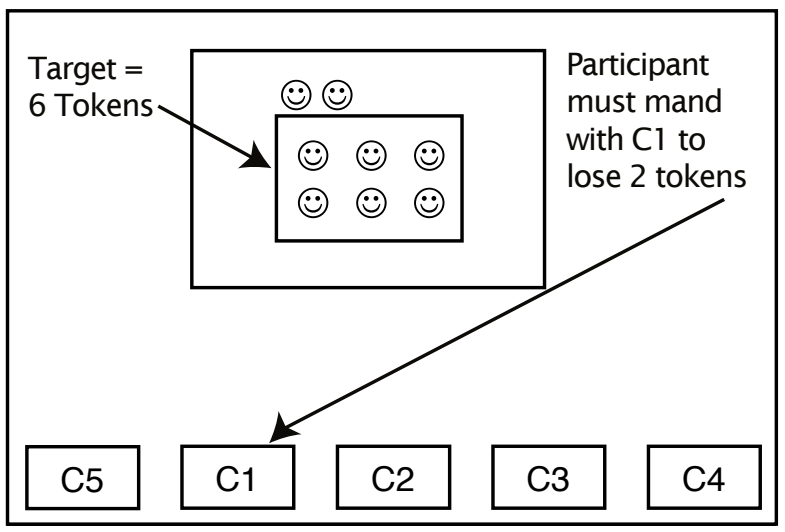

2C. Test II Example

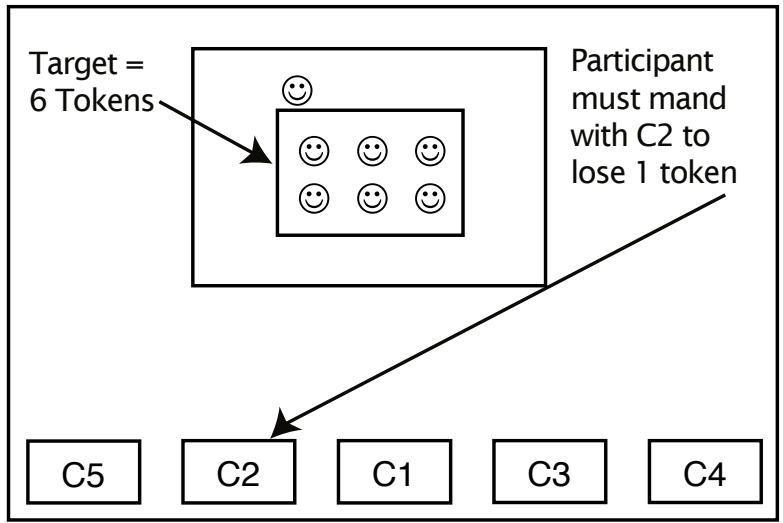

Figure $2 A, B, C$. Visual representation of board-game format used during mand training and testing. 
only the A1 or A2 samples, but after 4 consecutively correct trials the sample stimuli A3, A4, and A5 were gradually introduced into the quasi-random mix of trials with appropriate instructions (e.g., "Look here [A3] and point here [B3]"). To avoid unnecessary confusion for participants during these initial trials, the position of the B stimuli (comparisons) remained fixed. When all five samples and five comparisons had been introduced (approximately 10 trials), subsequent trials involved randomizing the position of the comparison stimuli. Only the latter trials were included when conditional discrimination training trials were counted in 25-trial blocks. The criterion for successful completion of A-B conditional discrimination training was a minimum of $22 / 25$ trials for two successive blocks. Presentation of samples was arranged in a quasi-random order, but each of the five A sample stimuli was presented five times within each block.

When participants had demonstrated a successful performance with A-B conditional discriminations, the investigator began training $\mathrm{B}-\mathrm{C}$ relations. The $\mathrm{B}-\mathrm{C}$ conditional discrimination training trials were conducted similarly as the A-B trials, except that the B stimuli were samples and the $\mathrm{C}$ stimuli were comparisons.

Test I: Derived transfer of five relational mand functions. When participants had successfully completed training in B-C conditional discriminations, they were exposed to a test for derived transfer of the five mand functions. The board-game format was used in a procedure that was similar to mand training, except for the following details.

The investigator took a seat to one side of the desk, and a parent replaced the investigator, sitting opposite the participant. During test trials the C stimuli were used in place of the A stimuli; participants were thus required to mand for $-2,-1,0,+1$, and +2 tokens, with $\mathrm{C} 1, \mathrm{C} 2, \mathrm{C} 3, \mathrm{C} 4$, and $\mathrm{C} 5$, respectively. During initial trials (3), participants were presented with three of the five $\mathrm{C}$ mand stimuli. One of the three $C$ stimuli was always the correct stimulus for manding appropriately. During the remaining trials, participants were presented with all five C stimuli (see Figure 2B). Neither the investigator nor the parent delivered programmed differential consequences for correct or incorrect mands during test trials. Instead, each time a participant manded, the investigator simply cleared the board and moved on to the next trial. The investigator delivered the following instructions to participants,

Like before, look at the board and count the tokens, then look carefully at the cards and give (Mummy/Daddy) the card you think is right. This time we won't give or take away tokens, and we won't tell you if you're right.

The criterion for successful completion of Test I was 25/25 correct responses or at least 18/20 for two successive blocks (including the three initial trials). The order of trial presentations was quasi-random, but participants were required to mand with each stimulus card $(\mathrm{C} 1, \mathrm{C} 2, \mathrm{C} 3, \mathrm{C} 4$, and $\mathrm{C} 5)$ at least five times. The investigator and the parent conducting the test both separately recorded each mand (nonsense syllable) presented by participants on a prepared score sheet.

Training reorganized conditional discriminations. Manipulation of conditional discrimination training functioned similarly as a return to baseline in a withdrawal design experiment. Thus training to reorganize conditional discriminations was followed by a test to determine whether the derived mands 
changed accordingly. Specifically, on the basis of the newly trained relations, it was expected that on this test occasion (Test II) participants would mand for -2 , $-1,0,+1$, and +2 , with C2, C1, C5, C3, and C4, respectively, in the absence of any direct training. Subsequently, the baseline conditional discriminations were retrained, and a test (Test III) probed for a return to the original derived mands. The expectation was that participants would once again show emergent manding for $-2,-1,0,+1$, and +2 , with $\mathrm{C} 1, \mathrm{C} 2, \mathrm{C} 3, \mathrm{C} 4$, and C5, respectively.

When the three participants had successfully completed Test I and demonstrated five derived mands, training commenced to reorganize the second set of conditional discriminations as follows: B1-C2, B2-C1, B3-C5, B4-C3, B5-C4. The procedure for reorganized conditional discrimination training was similar to the MTS training for the original B-C conditional discrimination training. Thus each of the "B" stimuli was rotated quasirandomly as a "sample" stimulus above the five "C" stimuli (comparisons). But on this occasion, differential reinforcement was provided for selecting the comparison $\mathrm{C} 2$ given the sample $\mathrm{B} 1$, selecting $\mathrm{C} 1$ given $\mathrm{B} 2$, selecting $\mathrm{C} 5$ given $\mathrm{B} 3$, selecting $\mathrm{C} 3$ given $\mathrm{B} 4$, and selecting $\mathrm{C} 4$ given $\mathrm{B} 5$. Initial instructions were provided (e.g., "Look here [B1], and point here [C2]") and faded as participants became proficient and pointed without instruction. The position of the five "C" stimuli was held constant across initial trials as all five conditional discriminations were gradually introduced. Only trials with all five $\mathrm{C}$ stimuli presented in random rotation were counted, and the training criterion was the same as the original training.

Test II: Five reorganized derived mand functions. The procedure for Test II was similar to that for Test I, except that participants were required to mand for, $-2,-1$, $0,+1$, and +2 tokens, with C2, C1, C5, C3, and C4, respectively (see Figure 2C).

Retraining original conditional discriminations. If participants successfully demonstrated five derived mand functions based on the novel conditional discriminations, they were exposed to training to reestablish the original $\mathrm{B}-\mathrm{C}$ conditional discriminations (B1-C1, B2-C2, B3-C3, B4-C4, and B5-C5). These conditional discriminations were trained exactly as before.

Test III: Return to original five derived mand functions. Test III was conducted exactly as Test I was.

\section{Results}

The data for training and testing for derived transfer of mand functions are presented in Figures 3-5. Ben (Figure 3) required minimal training to establish direct mands, and he achieved the criterion performance for both sets of conditional discriminations with minimal training (2 trial blocks). Subsequently, Ben successfully completed Test I, showing derived transfer of five relational mand functions. He again required minimal training during the training procedure to reorganize the conditional discriminations (B-C) but failed to show five reorganized derived mands when subsequently exposed to Test II. Ben underwent additional training (two blocks) in reorganized conditional discriminations, to strengthen the newly trained relations and facilitate reorganized derived manding. Subsequently, Ben was exposed to Test II on a second occasion and successfully demonstrated five reorganized derived mands. After minimal training to reestablish the baseline conditional discriminations, Ben successfully completed Test III, showing a return to the original five derived mands. 

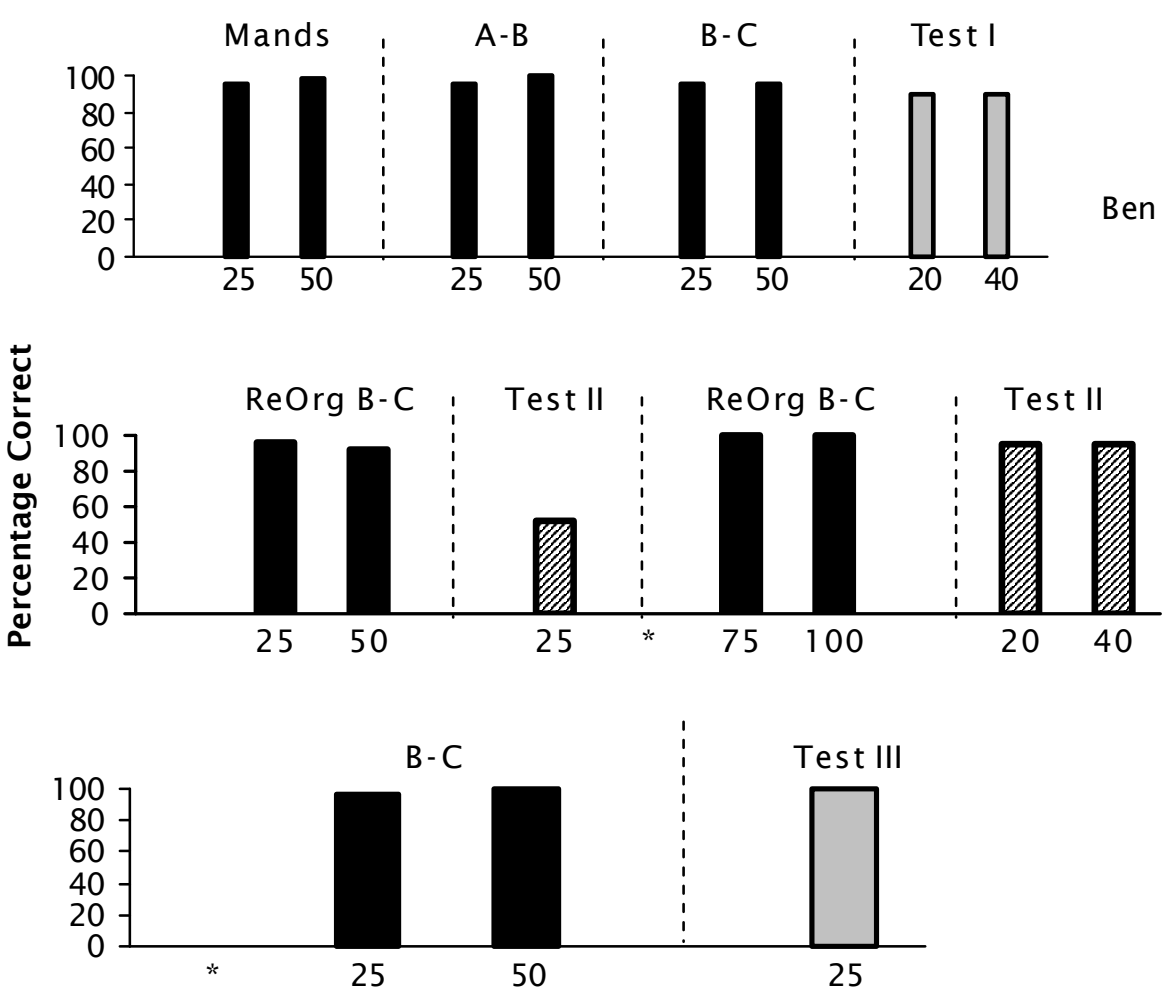

Blocks of Trials

Figure 3. Training and test results for Ben.

Jasmine (mild learning difficulties reported) achieved a criterion performance in manding and in both sets of conditional discriminations with minimal training. She proceeded to successfully complete Test I, demonstrating five derived mands (Figure 4). After minimal training to establish reorganized conditional discriminations, Jasmine went on to demonstrate the reorganized derived mands (Test II). She completed retraining in the baseline conditional discriminations but twice failed to demonstrate a return to the original derived mands (Test III) despite being exposed to additional retraining (two blocks). Experimental procedures were discontinued with Jasmine at this point for an interval of 3 weeks. After the break, Jasmine was exposed to the entire sequence involved in the baseline training (a refresher procedure), and she then completed Test III successfully.

The data for Shane (attending ABA programs for a reported speech delay) are presented in Figure 5. Like the other 2 participants, Shane required two trial blocks each for mand training and for both sets of conditional discrimination training. Subsequent to training procedures, Shane successfully completed Test I, demonstrating five derived mands. He completed training to reorganize the conditional discriminations, and then successfully completed Test II. Shane successfully completed retraining in the baseline conditional discriminations 

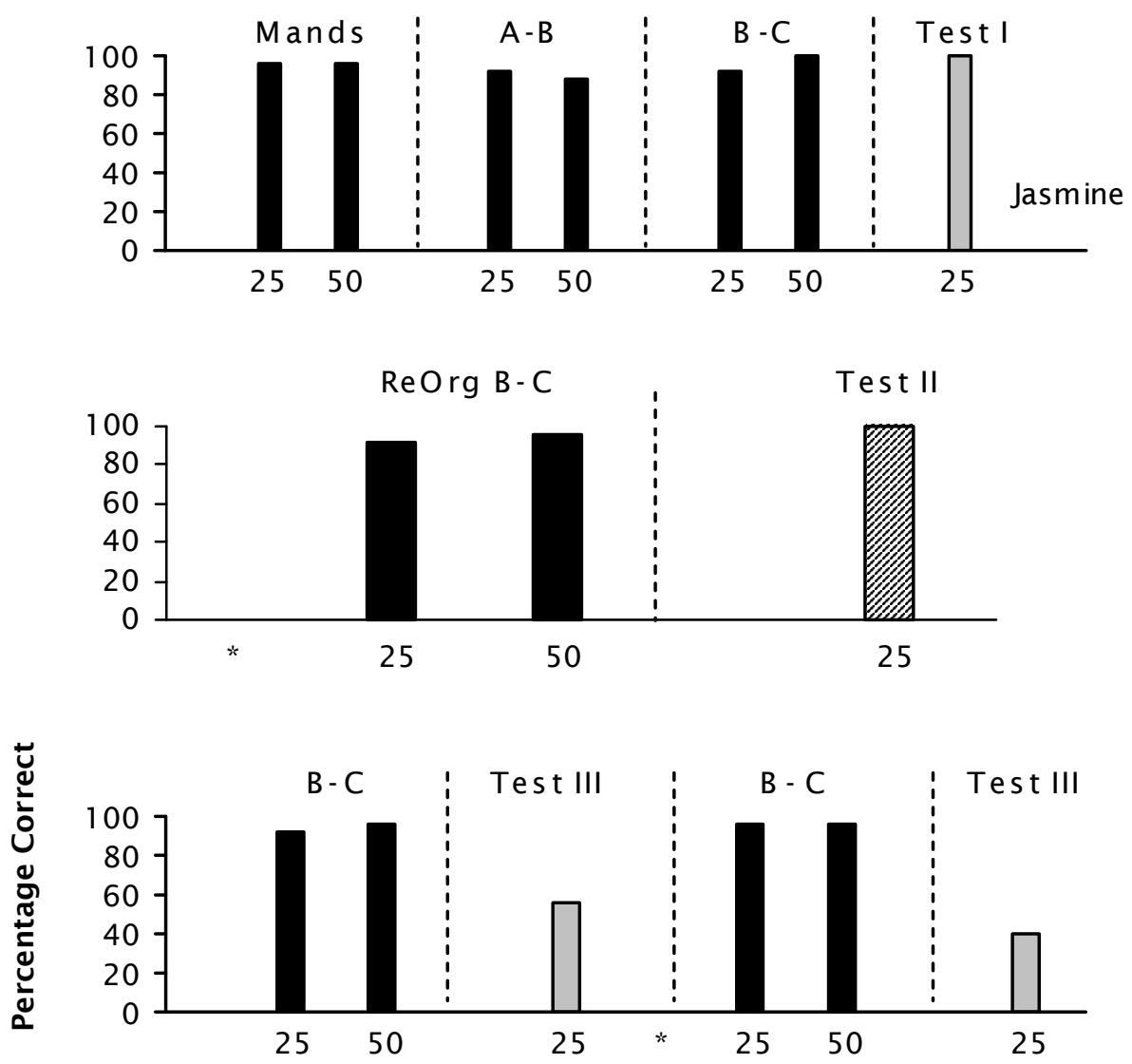

'R efresher' procedure - conducted after 3 week interval

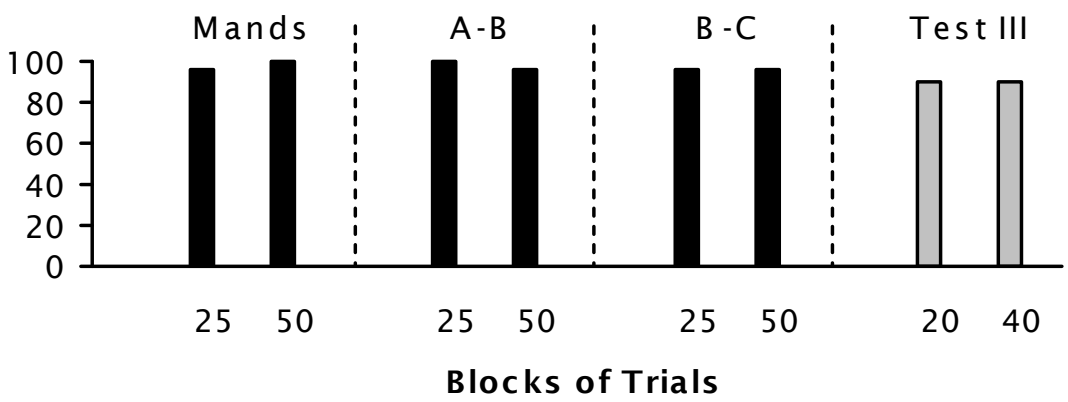

Figure 4. Training and test results for Jasmine.

but subsequently failed Test III. After additional retraining (two blocks) in the baseline conditional discriminations and a second exposure to Test III, Shane again failed the test, but his correct mands increased from $44 \%$ to $60 \%$. Similar to Jasmine, Shane was exposed to a "refresher" procedure after an interval of 3 weeks, and on this occasion he successfully completed Test III. 

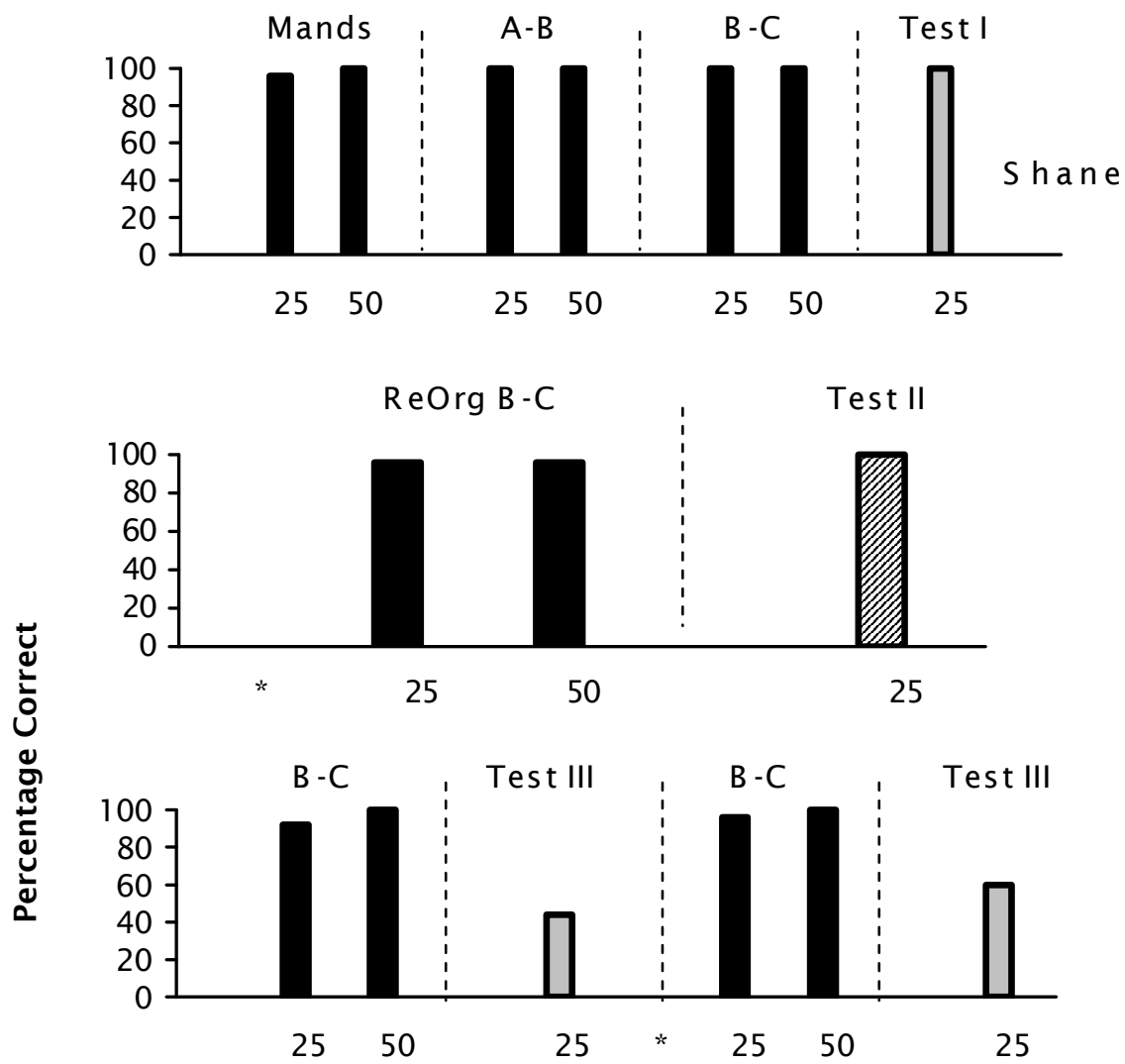

'R efresher' procedure - conducted after 3 week interval

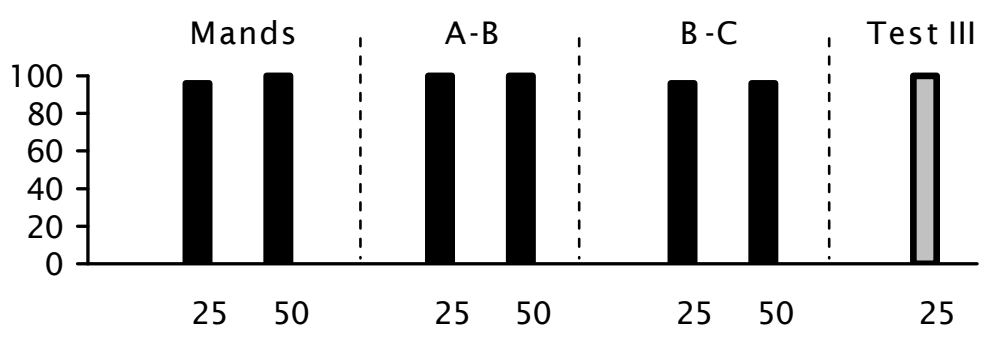

Blocks of Trials

Figure 5. Training and test results for Shane.

\section{General Discussion}

The 3 participants showed five derived mands and went on to show five reorganized derived relational mands and then a return to the original mands, in accordance with the trained conditional discriminations. 
Problems arose during Tests II and III. Ben initially failed Test II but was successful on a second occasion subsequent to additional training in the reorganized conditional discriminations. Two children with reported learning difficulties failed Test III on two occasions but successfully completed the test during a "refresher" procedure conducted after an interval of 3 weeks. The refresher procedure involved retraining the mands and baseline conditional discriminations and subsequent exposure to Test III. The difficulties with derived manding in accordance with reversal-type procedures are consistent with previous research on stimulus equivalence relations. Specifically, it has been reported that equivalence tests that followed altered or reversed contingencies in the trained conditional discriminations failed to generate appropriately modified equivalence relations (Pilgrim \& Galizio, 1990; Pilgrim \& Galizio, 1995; Pilgrim, Chambers, \& Galizio, 1995). Furthermore, the current study, consistent with our previous research, did not use MTS test procedures to probe for equivalence relations, because doing so may facilitate derived transfer based on direct associative processes, rather than transfer per se. Thus the difficulties observed in the current study in reversing previously derived performances are not unique. Nevertheless, the present research does show that it is possible to overcome difficulties related to derived transfer reversals.

The current study is the third in a series that has successfully combined Skinner's concept of a "mand" with RFT to establish increasingly complex derived manding (Murphy et al., 2005; Murphy \& Barnes-Holmes, in press). The two previous studies were conducted with children with autism, and the current study was conducted with 2 typically developing children and 1 child with a reported speech delay. Thus the strategies used appear to have the potential for use on a wide-ranging population, including those who are normally developing, as well as those with various types of developmental disabilities.

The current program of research has successfully set out clinical procedures for establishing what Skinner (1957) referred to as "magical mands," or mands that are emitted without a prior history of reinforcement. In addition, the research has shown a gradual development in the complexity of the novel mands that participants have shown. Briefly, the sequence has shown derived manding for additional single items, for the addition and removal of single items, and currently, for the addition and removal of specific numbers of items.

Establishing complex derived manding could be seen as training or teaching higher "cognitive" processes with children in whom, for example, autism has been diagnosed. Given that children with autism are said to have less flexible cognitive repertoires (Wahlberg \& Jordan, 2001) and may not readily show emergent manding (Hall \& Sundberg, 1987; Lamarre \& Holland, 1985; Nuzzolo-Gomez \& Greer, 2004; Petursdottir et al., 2004; Sigafoos et al., 1990; Sundberg et al., 1990; Twyman, 1996), the development of procedures to establish complex derived mand repertoires seems important. In terms of practical utility, the procedures developed in the current study could be adapted for children with autism who rigidly apply just one particular mand form. Before attempting to establish derived manding for specific amounts, however, it may be important to ensure that the children are able to add and subtract single digits up to the level of the desired derived manding, as this may impact on the derived performance. 
Practical application of procedures might be arranged as follows. First, MTS training using two sets of printed-word stimuli might be used to establish conditional discriminations (A1-B1-C1-D1). Each stimulus could consist of a particular printed "give me"' mand for the addition of specific amounts. "I

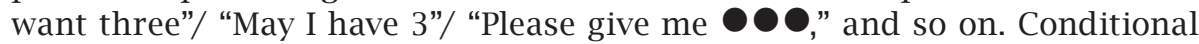
discrimination training would then be followed by a mand procedure during which three tokens are absent on a board. The three tokens would be delivered contingent on the child uttering an appropriate mand, and reinforcement delivered contingent on obtaining the correct number of tokens on the board. If a child uses a particular mand form twice consecutively (e.g., "Can I have three please?"), the child would be prompted to "ask a different way," and the token would be delivered only when the child used a different mand form. The same procedure could then be used to establish multiple mand forms for requesting the addition and, with appropriate modification, the removal of specific numbers of tokens. Such training may thus establish greater verbal flexibility in an otherwise rigid behavioral repertoire (Neuringer, 2004; Neuringer, Deiss, \& Olson, 2000; Pryor, Haag, \& O’Reilly, 1969).

\section{References}

BARNES, D., BROWNE, M., SMEETS, P., \& ROCHE, B. (1995). A transfer of functions and a conditional transfer of functions through equivalence relations in three- to six-year-old children. The Psychological Record, 45, 405-430.

BARNES-HOLMES, D., BARNES-HOMES, Y., \& CULLINAN, V. (2000). Relational frame theory and Skinner's Verbal Behavior: A possible synthesis. The Behavior Analyst, 23, 69-84.

CHOMSKY, N. (1959). A review of Verbal Behavior by B. F. Skinner. Language, $35,26-58$.

DOUGHER, M. J., AUGUSTON, E., MARKHAM, M. R., GREENWAY, D. E., \& WULFERT, E. (1994). The transformation of eliciting functions through stimulus equivalence classes. Journal of the Experimental Analysis of Behavior, 62, 331-351.

HALL, G., \& SUNDBERG, M. L. (1987). Teaching mands by manipulating conditioned establishing operations. The Analysis of Verbal Behavior, 5, 41-53.

HAYES, S. C., BARNES-HOLMES, D., \& ROCHE, B. (2001). Relational frame theory: A post-Skinnerian account of language and cognition. New York: Plenum.

LAMARRE, J., \& HOLLAND, J. (1985). The functional independence of mands and tacts. Journal of the Experimental Analysis of Behavior, 43, 5-19.

LARAWAY, S., SNYCERSKI, S., MICHAEL, J., \& POLING, A. (2003). Motivating operations and terms to describe them: Some further refinements. Journal of Applied Behavior Analysis, 36, 407-414.

LERMAN, D. C., PARTEN, M., ADDISON, M. R., VORNDREN C. M., VOLKERT, V. M., \& KODAK, T. (2005). A methodology for assessing the functions of emerging speech in children with developmental disabilities. Journal of Applied Behavior Analysis, 38, 303-316.

MICHAEL, J. (1993). Establishing operations. Behavior Analyst, 16, 191-206.

MURPHY, C., BARNES-HOLMES, D., \& BARNES-HOLMES, Y. (2005). Derived manding with seven children diagnosed with autism: Synthesizing Skinner's Verbal Behavior with relational frame theory. Journal of Applied Behavior Analysis, 38, 445-462. 
MURPHY, C., \& BARNES-HOLMES, D. (in press). Derived more/less relational mands with three children diagnosed with autism: Synthesizing Skinner's Verbal Behavior with relational frame theory II. Journal of Applied Behavior Analysis.

NEURINGER, A. (2004). Reinforced variability in animals and people: Implications for adaptive action. American Psychologist, 59, 891-906.

NEURINGER, A., DEISS, C., \& OLSON, G. (2000). Reinforced variability and operant learning. Journal of Experimental Psychology: Animal Behavior Processes, 26, 79-94.

NUZZOLO-GOMEZ, R., \& GREER, R. D. (2004). Emergence of untaught mands or tacts of novel adjective-object pairs as a function of instructional history. The Analysis of Verbal Behavior, 20, 63-76.

PETURSDOTTIR, A. I., CARR, J. E., \& MICHAEL, J. (2005). Emergence of mands and tacts of novel objects among preschool children. The Analysis of Verbal Behavior, 21, 59-74.

PILGRIM, C., CHAMBERS, L., \& GALIZIO, M. (1995). Reversal of baseline relations and stimulus equivalence: II. Children. Journal of the Experimental Analysis of Behavior, 63, 239-254.

PILGRIM, C., \& GALIZIO, M. (1990). Relations between baseline contingencies and equivalence probe performances. Journal of the Experimental Analysis of Behavior, 54, 213-224.

PILGRIM, C., \& GALIZIO, M. (1995). Reversal of baseline relations and stimulus equivalence: I. Adults. Journal of the Experimental Analysis of Behavior, 63, 225-238.

PRYOR, K. W., HAAG, R., \& O'REILLY, J. (1969). The creative porpoise: Training for novel behaviour. Journal of Experimental Analysis of Behavior, 12, 653-661.

REHFELDT, R. A., \& ROOT, S. L. (2005). Establishing derived requesting skills in adults with severe developmental disabilities. Journal of Applied Behavior Analysis, 38, 101-105.

ROSALES, R., \& REHFELDT, R. A. (2007). Contriving transitive conditioned establishing operations to establish derived manding skills in adults with severe developmental disabilities. Journal of Applied Behavior Analysis, 40, 105-121.

SIGAFOOS, J., REICHLE, J., DOSS, S., HALL, K., \& PETTITT, L. (1990). "Spontaneous" transfer of stimulus control from tact to mand contingencies. Research in Developmental Disabilities, 11, 165-176.

SKINNER, B. F. (1957). Verbal behavior. Englewood Cliffs, NJ: Prentice Hall. SMEETS, P. M., \& STRIEFEL, S. (1994). A revised block-trial procedure for establishing arbitrary matching in children. The Quarterly Journal of Experimental Psychology, 47B, 241-261.

SUNDBERG, M. L., \& MICHAEL, J. (2001). The benefits of Skinner's analysis of verbal behavior for children with autism. Behavior Modification, 5, 698-724.

SUNDBERG, M. L., \& PARTINGTON, J. W. (1998). The Assessment of Basic Language and Learning Skills (The ABLLS): An assessment, curriculum guide, and skills tracking system for children with autism or other developmental disabilities. Pleasant Hill, CA: Behavioral Analysts, Inc.

SUNDBERG, M. L., SAN JUAN, B., DAWDY, M., \& ARGUELLES, M. (1990). The acquisition of tacts, mands, and intraverbals by individuals with traumatic brain injury. The Analysis of Verbal Behavior, 8, 83-99. 
TWYMAN, J. (1996). The functional independence of impure mands and tacts of abstract stimulus properties. The Analysis of Verbal Behavior, 13, $1-19$.

WAHLBERG, T., \& JORDAN, S., (2001). A case study in the dynamics of autism. In T. Wahlberg, F. E. Obiakor, \& S. Burkhardt (Eds.), Autistic spectrum disorders: Educational and clinical interventions (pp. 53-65). Oxford, UK: Elsevier. 
